\title{
Subcellular localisation of radionuclides by transmission electronic microscopy: Application to uranium, selenium and aquatic organisms
}

\author{
M. Floriani \\ Laboratoire de Radioécologie et d'Écotoxicologie, Institut de Radioprotection et de Sûreté \\ Nucleaire, DEI/SECRE/LRE, Cadarache, Bât. 186, BP. 3, \\ 13115 St-Paul-Lez-Durance Cedex, France
}

\begin{abstract}
The global framework of this study is to go further in the understanding of the involved mechanisms of uranium and selenium internalisation at the subcellular level and of their toxicity towards several aquatic organisms. In this context, the applications and performances of a Scanning Transmission Electronic Microscope (STEM), which is fitted with a CCD camera and an Energy-Dispersive-X-Ray (EDX) analysis are reported. This equipment provides a direct correlation between a histological image (0.34 nm resolution) and a clear expression of element distribution. Demonstration of the usefulness of this method to understand the bioaccumulation mechanisms and to study the effect of the pollutant uptake at the subcellular level has been performed for target organs of a metal (U) and a metalloid (Se) in various biological models: a freshwater crayfish (Orconectes Limosus) and a unicellular green alga (Chlamydomonas reinhardtii)). TEM-EDX analysis revealed the presence of U-deposits in gills and digestive gland in crayfish. In the alga, the accumulation of Se was found in electron-dense granules within the chloroplast with ultrastructural changes and starch accumulation.
\end{abstract}

\section{INTRODUCTION: DESCRIPTION AND PRINCIPLE OF THE SCANNING TRANSMISSION ELECTRONIC MICROSCOPE}

This Scanning Tecnai G ${ }^{2} 12$ (FEI) microscope is configurated for high contrast with the BioTwin lens configuration. To facilitate digital capture, a CCD camera (SIS Megaview III) has been integrated in the microscope with an Energy Dispersive X-ray detector-SUTW (EDAX-Phoenix) to characterise the chemical composition of targeted structures.

The TEM/STEM is an evacuated metal cylinder (the column) about 2 meters high with the source of illumination, a Lab6 filament (the cathode), at the top (figure 1). If the filament is heated and a high voltage (the accelerating voltage) of between 20,000 to 120,000 volts is passed between it and the anode, the filament will emit electrons. These negatively charged electrons are accelerated to an anode (positive charge) placed just below the filament, some of which pass through a tiny hole in the anode, to form an electron beam, which passes down the column. The speed at which they are accelerated to the anode depends on the amount of accelerating voltage present. Electro-magnets, placed at intervals down the column, focus the electrons. The double condenser lenses focus the electron beam onto the specimen, which is placed on a copper grid. As the electron beam passes through the specimen, some electrons are scattered whilst the remainder are focused by the objective lens onto a phosphorescent screen to form an image. Unfocused electrons are blocked out by the objective aperture, resulting in an enhancement of the image contrast. The contrast of the image can be increased by reducing the size of this aperture. The remaining lenses on the TEM are the intermediate lens and the projector lens. The intermediate lens is used to control magnification. 
The projector lens forms a real image on the fluorescent screen at the base of the microscope column. Photos taken with electron microscopes are always black and white. The degree of darkness corresponds to the electron density (= differences in atom masses) of the candled preparation.

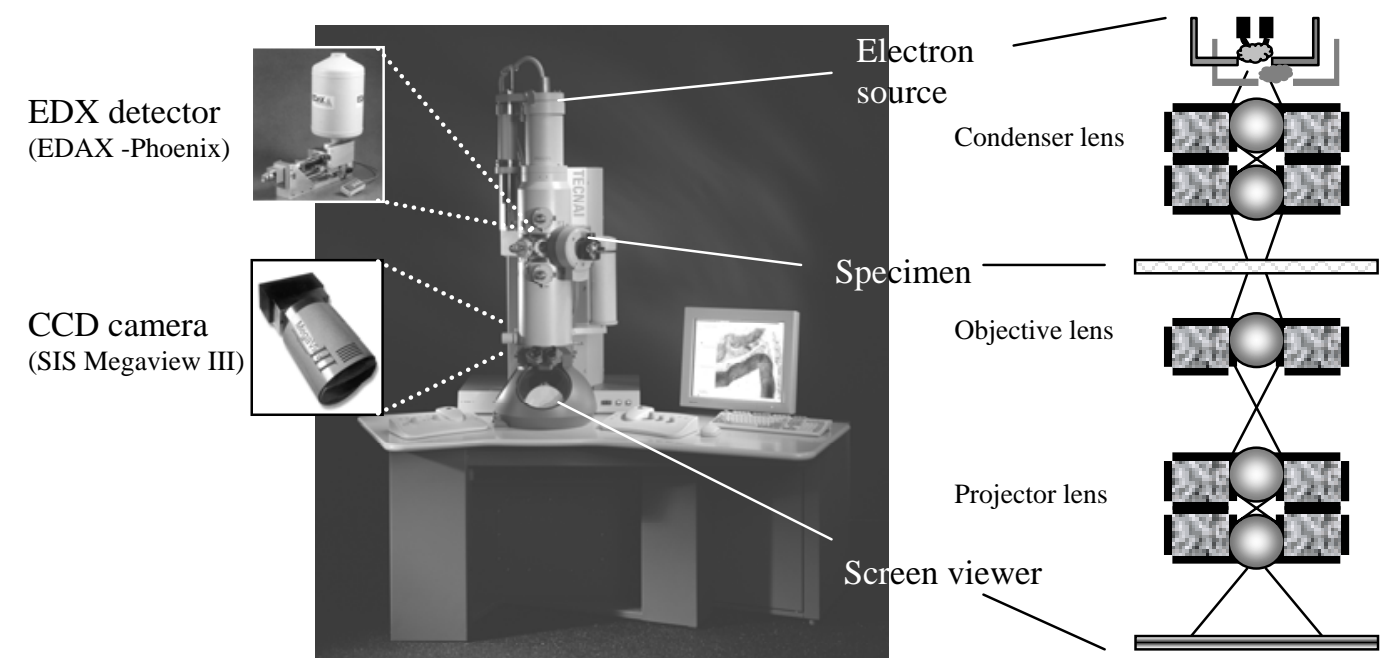

Figure 1. Schema of the Tecnai $\mathrm{G}^{2} 12$ Biotwin Microscope (FEI).

\section{SPECIMEN PREPARATION FOR TEM/STEM}

The greatest obstacle to examining biological material with the electron microscope is the unphysiological conditions to which specimens must be exposed. First the biological specimen must be fixed so that its ultrastructure is as close to that in the living material when exposed to the very high vacuum $\left(10^{-5}\right.$ to $10^{-8}$ Torr $)$. Moreover, the limited penetrating power of electrons means that the specimens must be sliced into ultrathin sections to allow electrons to pass through. Last, contrast in the TEM depends on the atomic number of the atoms in the specimen; the higher the atomic number, the more electrons are scattered and the greater the contrast. Since biologicals specimens are composed mostly of atoms of low atomic number ( $, \mathrm{H}, \mathrm{O}, \mathrm{N})$, ultrathin sections are made visible by selective staining which are electron opaque. The basic procedure for the preparation of biological samples for the TEM is as follows and illustrated in figure 2:

1 Dissection of organs into $1 \mathrm{~mm}^{3}$,

2 Fixation of the tissue with $1.5 \%$ glutaraldehyde in sodium cacodylate buffer $(0.1 \mathrm{M}, \mathrm{pH} 7.4)$ (to stop cellular processes as rapidly as possible and cross-links cellular structures to preserve their "normal" morphology and chemical composition),

3 Postfixation with $1 \%$ osmium tetroxyde in the same cacodylate buffer,

4 Dehydration of the specimen in a grades series of ethanol,

5 Embeddment: Once the sample is completely infiltrated by epoxy resin, it is placed in a mold and polymerised at $60^{\circ} \mathrm{C}$. The mold is usually chosen to accommodate the particular type of sample (flat embeddment, pellet of cells, etc.) and so that the finished block will easily fit into the ultramicrotome,

6 Sectioning ultrathin $(70-140 \mathrm{~nm})$ or semithin $(200-500 \mathrm{~nm})$ sections on copper grid from the polymerized block by using an ultramicrotome (Leica) with a diamond knife (Diatome),

7 Staining for adding contrast to the specimen elements (exposure to salts of uranium and/or lead). 


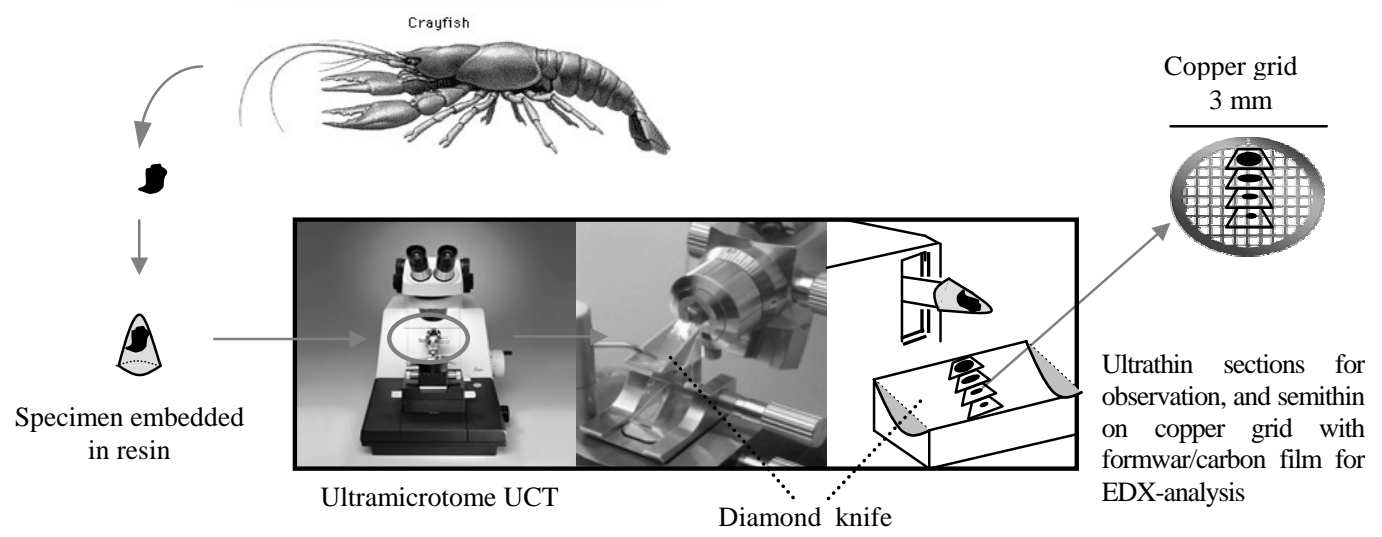

Figure 2. Basic procedure for the preparation of biological samples for the TEM.

\section{PHOTO GALLERY}

Figure 3 shows some photos taken by the Tecnai 12 Biotwin STEM which has a resolution of $0.344 \mathrm{~nm} /$ line and $0.500 \mathrm{~nm} /$ point with a magnification power of 300000 times.
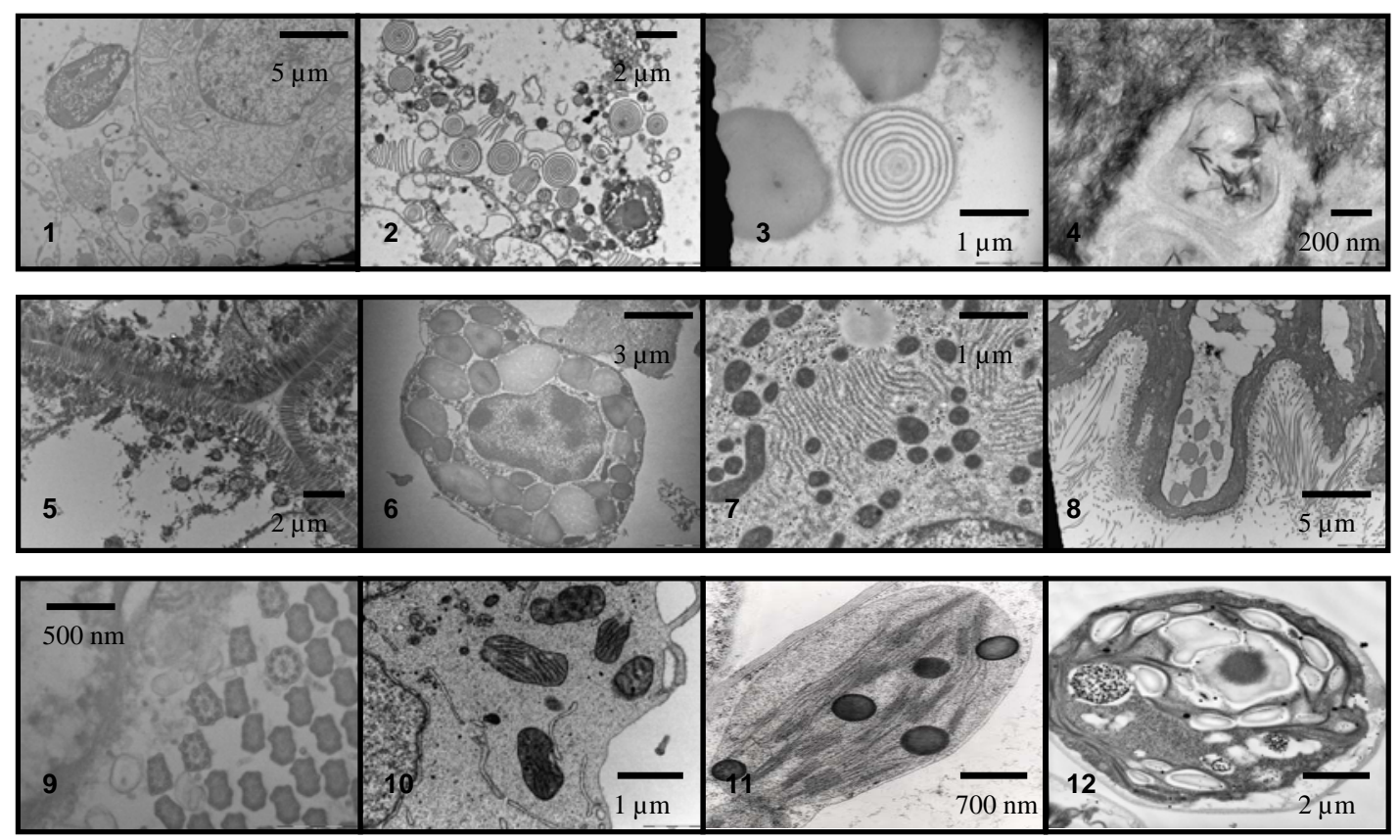

Figure 3. 1) Gills epithelium of Orconectes limosus , 2-3) Spherocristal of mineral accumulation, 4) Uranium precipitates, 5) Microvillosity of digestive gland of Orconectes limosus, 6) Cell of digestive gland of Orconectes limosus, 7) Rough endoplasmatic reticulum, 8) Gills filaments of Corbicula fluminea, 9) Cross section of lateral cilium of Corbicula fluminea, 10) Cytoplasma with mitochondria, 11) Chloroplast of scape of Phaseolus vulgaris, 12) Chlamydomonas reinhardtii. 


\section{X-RAY ANALYSIS}

When the beam interacts with the sample, energy is released in the form of radiation over a variety of wavelengths (figure 4). This energy results from beam electrons ionising the inner electron shells of an atom. When the excited atom relaxes, i.e., an outer electron filling the inner shell, radiation is released which is characteristic of the atom electron shells' energy levels. We speak of these events as either K, L, or M replacement events, depending on which orbital shell lost the electron. The portion of radiation in the X-Ray part of the spectrum are referred to as Characteristic X-rays.

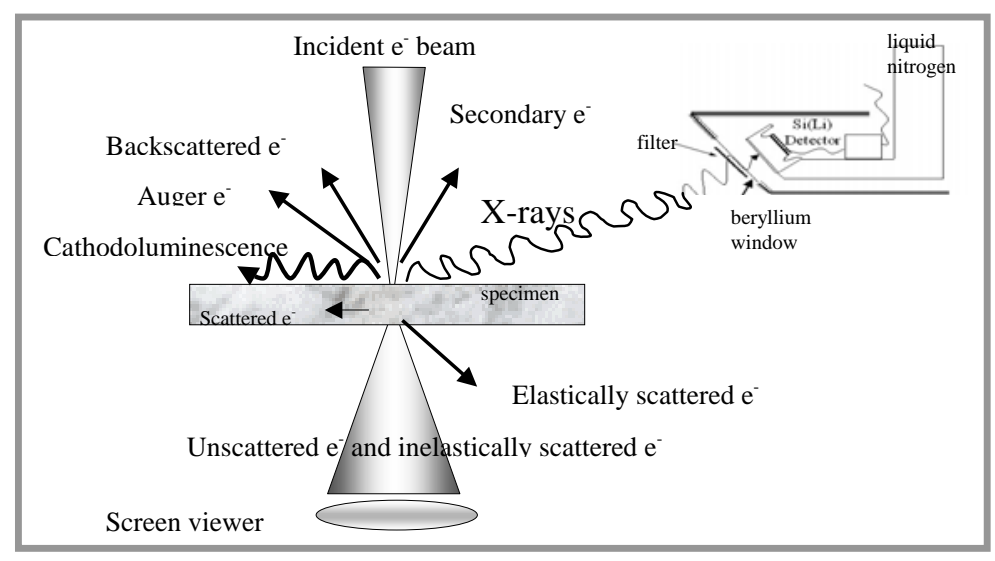

Figure 4. Electron beam/specimen interactions.

The X-rays generated are collected and plotted as a spectrum (figure $5 \mathrm{~b}$,d and figure $6 \mathrm{~d}$ ) or a map by an EDX-detector. Each peak on the spectrum represents a transition with a characteristic energy. By collecting the x-ray signals produced over an extended period of time (e.g., 100 seconds) certain electron replacement events will occur more frequently than others. We collect the signals as long as the most frequent events will reinforce each other and thereby become distinct from the background and continuum x-rays. These repeated energy spectra manifest themselves in the form of distinct peaks.

With a STEM, it is possible to obtain a precise elemental composition of a biological sample with high spatial resolution and from areas as small as 1 to $50 \mathrm{~nm}$ in diameter. The reason is that a STEM is equipped with a set of scan coils and can function in much the same way as an SEM by reducing the beam to a small spot over the specimen which in this case would be a section on a grid. For instance, if a plant cell is found to have an electron dense inclusion of unknown composition. By bombarding the inclusion with electrons from the beam, we drive off a number of electrons, which are replaced by outer orbital electrons and give off characteristic x-rays for the elements in the specimen. Since we are primarily interested in the composition of the inclusion and not the surrounding tissue, it is very beneficial to be able to focus the beam onto a single spot and position it over the object of interest. This can best be done in a STEM.

The EDX-detector can measure nearly the entire spectrum of the element (starting from Boron) and the detection sensitivity is very dependent on the molecular weight of the element ( $1 \%$ for boron; $0.1 \%$ for carbon and $0.01 \%$ for uranium). It is possible to calculate absolute concentrations of individual elements with appropriate corrections by comparing spectra with others collected from samples of known composition.

One can also establish simultaneous distribution maps of 26 elements, and then do colour mixings. Quantitative analysis can next be applied to images in order to know the percentage of distribution of surfaces, the number of grains, the perimeters, etc. 


\section{SUBCELLULAR LOCALISATION OF URANIUM IN THE FRESHWATER CRAYFISH ORCONECTES LIMOSUS}

According to measurements of uranium organs distribution, the gills and digestive gland of crayfish are the target-organs of the uranium bioaccumulation. That is why STEM-EDX studies are focused on these organs [Envirhom, 2003]. As illustrated in figure 5, uranium appears to be mostly precipitated in a biofilm (algae and bacteria) outside the gill and, to a lesser extent, within the gill epithelium. First observations show that uranium can only be found at the extremities of the digestive gland microvillosities and not inside the digestive gland epithelium (not illustrated). Studies are being conducted so as to confirm these results.
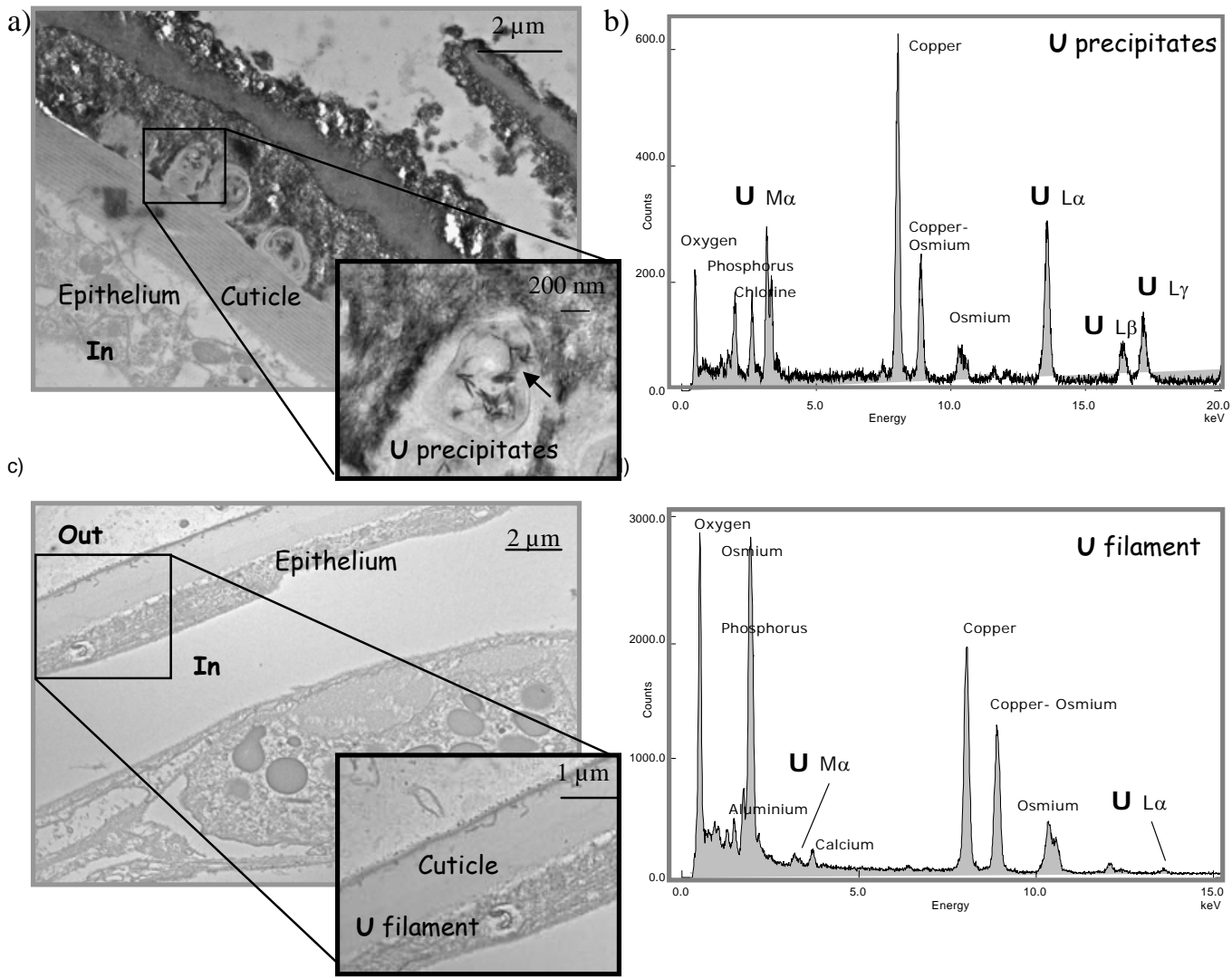

Figure 5. a) Photo of U precipitates outside the gill of Orconectes limosus; b) EDX spectrum of U-precipitates; c) Photo of U filament inside the gill of Orconectes limosus; d) EDX spectrum of U-filament.

\section{SUBCELLULAR LOCALISATION OF SELENIUM IN THE GREEN ALGA CHLAMYDOMONAS REINHARDTII}

As can be seen in figure 6, there is an appearance of electron-dense granules within the chloroplast associated with ultrastructural changes of chloroplasts (pyrenoids, thylakoids and stroma) and an increase of starch grains, in cells exposed to selenite. EDX-analysis revealed that these deposits are rich in selenium and also in phosphorus [Morlon, 2004]. 
a)

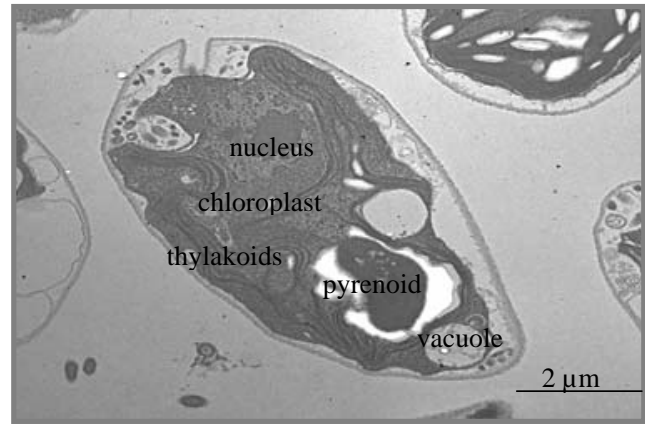

c)

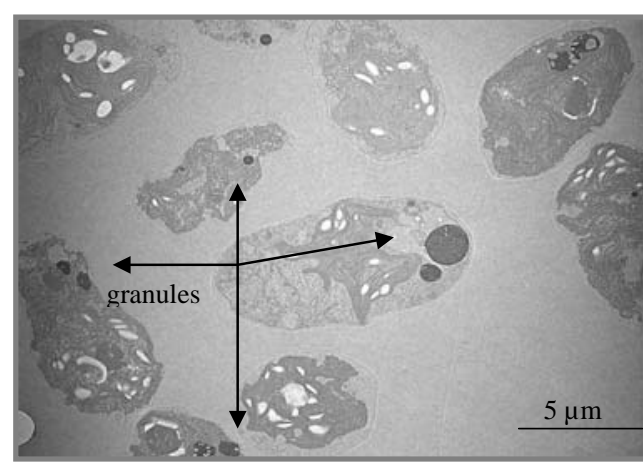

b)

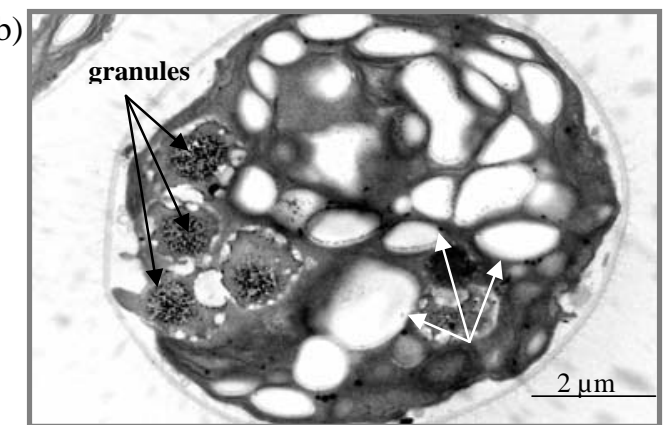

d)

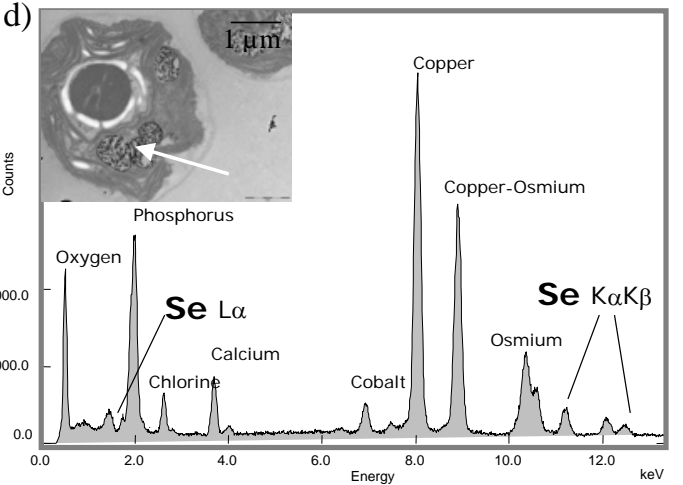

Figure 6. Photos of Chlamydomonas reinhardtii; a) Non-exposed alga; (b,c,d) Alga exposed to selenite; d) EDX-spectrum of Se-aggregates inside the chloroplaste.

\section{CONCLUSION}

The TEM technique coupled with an EDX analysis can give, simultaneously, information on the histological structures of the studied organisms, the subcellular localisation of the different elements and their chemical characterisation. Thanks to this device we have revealed the presence of U-deposits in gills (inside and outside the cuticle) and the digestive gland of crayfish. In the alga, the accumulation of Se was found in electron-dense aggregates within the chloroplast associated with ultrastructural changes and starch accumulation. Therefore, the TEM-EDX should be considered as a major tool for performing bioaccumulation studies.

\section{Acknowledgments}

This work is a part of the ENVIRHOM program funded by the Institute for Radioprotection and Nuclear Safety.

\section{References}

[1] Glauert A.M. and Lewis P.R., Biological Specimen Preparation for Transmission Electron Microscopy (Princeton Univ Pr, 1998) Volume 17.

[2] Morgan A.J., X-ray Microanalysis in Electron Microscopy for Biologists (Oxford University Press, Royal Microscopical Society, 1985).

[3] Morlon H., Fortin C., Floriani M., Adam C., Garnier-Laplace J. and Boudou A., Aquat. Toxicol. (Submitted).

[4] Envirhom (2003). Bioaccumulation of radionuclides in situations of chronic exposure of ecosystems and members of the public, Progress Report 1, Technical Notes (Vol 2), DEI/03-01 \& DRPH/03-03. 